\title{
Autoimmune hepatitis in 847 childhood- onset systemic lupus erythematosus population: a multicentric cohort study
}

\author{
Verena A. Balbi ${ }^{1 \dagger}$, Bárbara Montenegro ${ }^{1 \dagger}$, Ana C. Pitta ${ }^{1 \dagger}$, Ana R. Schmidt ${ }^{1}$, Sylvia C. Farhat ${ }^{1}$, Laila P. Coelho ${ }^{1}$,
} Juliana C. O. Ferreira ${ }^{1}$, Rosa M. R. Pereira ${ }^{2}$, Maria T. Terreri ${ }^{3}$, Claudia Saad-Magalhães ${ }^{4}$, Nadia E. Aikawa ${ }^{2}$, Ana P. Sakamoto ${ }^{3}$, Kátia Kozu ${ }^{1}$, Lucia M. Campos ${ }^{1}$, Adriana M. Sallum ${ }^{1}$, Virginia P. Ferriani ${ }^{5}$, Daniela P. Piotto ${ }^{3}$, Eloisa Bonfá $^{2 \dagger}$ and Clovis A. Silva ${ }^{1,2^{*}+}$ (D)

\begin{abstract}
Objective: To evaluate autoimmune hepatitis (AIH) in a multicenter cohort of childhood-onset systemic lupus erythematosus (CSLE) patients.

Methods: This retrospective multicenter study included 847 patients with cSLE, performed in 10 Pediatric Rheumatology services of São Paulo state, Brazil. AlH was defined according to the International Autoimmune Hepatitis Group criteria (IAHGC). The statistical analysis was performed using the Bonferroni's correction $(p<0.0033)$.

Results: AlH in CSLE patients confirmed by biopsy was observed in 7/847 (0.8\%) and all were diagnosed during adolescence. The majority occurred before or at cSLE diagnosis [5/7 (71\%)]. Antinuclear antibodies were a universal finding, 43\% had concomitantly anti-smooth muscle antibodies and all were seronegative for anti-liver kidney microsomal antibodies. All patients with follow-up $\geq 18$ months (4/7) had complete response to therapy according to IAHGC. None had severe hepatic manifestations such as hepatic failure, portal hypertension and cirrhosis at presentation or follow-up. Further comparison of 7 CSLE patients with $\mathrm{AlH}$ and 28 without this complication with same disease duration [0 (0-8.5) vs. $0.12(0-8.5)$ years, $p=0.06$ revealed that the frequency of hepatomegaly was significantly higher in cSLE patients in the former group ( $71 \%$ vs. $11 \%, p=0.003$ ) with a similar median SLEDAl-2 K score [6 (0-26) vs. $7(0-41), p=0.755]$. No differences were evidenced regarding constitutional involvement, splenomegaly, serositis, musculoskeletal, neuropsychiatric and renal involvements, and treatments in CSLE patients with and without AIH $(p>0.0033)$.
\end{abstract}

Conclusions: Overlap of AlH and CSLE was rarely observed in this large multicenter study and hepatomegaly was the distinctive clinical feature of these patients. AlH occurred during adolescence, mainly at the first years of lupus and it was associated with mild liver manifestations.

Keywords: Autoimmune hepatitis, Childhood systemic lupus erythematosus, Hepatomegaly, Multicenter study

\footnotetext{
* Correspondence: clovis.silva@hc.fm.usp.br

†Verena A. Balbi, Bárbara Montenegro, Ana C. Pitta, Eloisa Bonfá and Clovis A.

Silva contributed equally to this work.

${ }^{1}$ Pediatric Rheumatology Unit, Children's Institute, Hospital das Clinicas

HCFMUSP, Faculdade de Medicina, Universidade de Sao Paulo, Sao Paulo, SP,

Brazil

${ }^{2}$ Division of Rheumatology, Hospital das Clinicas HCFMUSP, Faculdade de

Medicina, Universidade de Sao Paulo, Sao Paulo, SP, Brazil

Full list of author information is available at the end of the article
}

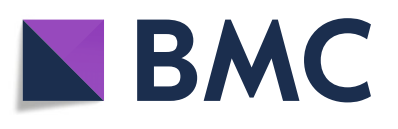

(c) The Author(s). 2018 Open Access This article is distributed under the terms of the Creative Commons Attribution 4.0 International License (http://creativecommons.org/licenses/by/4.0/), which permits unrestricted use, distribution, and

reproduction in any medium, provided you give appropriate credit to the original author(s) and the source, provide a link to the Creative Commons license, and indicate if changes were made. The Creative Commons Public Domain Dedication waiver (http://creativecommons.org/publicdomain/zero/1.0/) applies to the data made available in this article, unless otherwise stated. 


\section{Introduction}

Childhood-onset systemic lupus erythematosus (cSLE) is an autoimmune and inflammatory disease that affects multiple organs and systems, including liver [1-3].

Of note, autoimmune hepatitis $(\mathrm{AIH})$ is characterized by elevated hepatic enzymes, hypergammaglobulinemia, presence of autoantibodies and liver histology abnormalities, particularly interface hepatitis and lymphocytic infiltrates $[4,5]$. To our knowledge the prevalence of overlap $\mathrm{AIH}$ and cSLE in a large population was not studied and analysis of this very rare association is restricted to few case reports or case series [1, 3, 6, 7].

Therefore, the objective of this multicenter cohort study was to evaluate cSLE and AIH and the possible association with demographic data, cumulative clinical manifestations, treatments and outcomes in a large cSLE population in Latin America.

\section{Methods}

This study was conducted in 10 Pediatric Rheumatology services in the state of São Paulo, Brazil including a population of 847 cSLE patients [8]. All patients fulfilled the American College of Rheumatology (ACR) criteria for SLE [9], with disease onset before the age of 18. The Ethical Committee of each University Hospital approved this study. An investigator meeting was held for this study in São Paulo city to define the protocol according to the clinical parameters and disease activity tool scoring. At least one investigator with Brazilian Board Pediatric Rheumatology Certifying Examination supervised data collection in each center, reviewed paper files and tried to solve discrepancies among investigators of these centers. Four rounds of queries were performed to check for accuracy and sort out discrepancies among groups.

Patients' medical charts were systematically reviewed according to demographic data, clinical features and $\mathrm{AIH}$ characteristics, laboratorial abnormalities, therapeutic data and outcomes. AIH was diagnosed according to International Autoimmune Hepatitis Group criteria (IAHGC) $[4,5]$. Every medical visit from cSLE diagnosis to last visit or death was reviewed in each center. Percutaneous needle liver biopsy was performed in these centers in cSLE patients with elevation of liver enzymes (not related to hepatotoxic drugs, metabolic disease, alcohol or viral disease), associated with hypergammaglobulinemia and presence of at least one autoantibody [antinuclear antibodies (ANA), anti-type I liver-kidney microsomal (anti-LKM-1) antibody or liver and stomach tissue substrates and anti-smooth muscle antibody (anti-SMA)].

Descriptors of SLE Disease Activity Index 2000 (SLEDAI-2 K) were used to define clinical manifestations [10], and custom definitions as previously reported [8]. Cumulative clinical manifestations included constitutional involvement [defined as fever and lymphadenopathy (peripheral lymph node enlargement $>1.0 \mathrm{~cm}$ )], hepatomegaly [based on physical exam with liver edge $\geq 2 \mathrm{~cm}$ below the right costal margin or imaging (ultrasound or computer tomography when available)], splenomegaly [based on physical exam with palpable spleen or imaging (ultrasound or computer tomography when available)], musculoskeletal involvement, serositis, neuropsychiatric and renal involvement. Neuropsychiatric Lupus included 19 syndromes according to ACR classification criteria [11].

Antinuclear antibodies (ANA) were tested by indirect immunofluorescence. Anti-type I liver-kidney microsomal (anti-LKM-1) antibody on frozen sections of rodent kidney, liver and stomach tissue substrates and anti-smooth muscle antibody (anti-SMA) by indirect immunofluorescence on rat liver and kidney tissue sections on frozen sections of rodent kidney, liver and stomach tissue substrates. The cutoff values from the kit manufacturer were used to define abnormal.

Drug treatment data (prednisone, intravenous methylprednisolone, chloroquine diphosphate, hydroxychloroquine sulfate, methotrexate, azathioprine, cyclosporine, mycophenolate mofetil, intravenous cyclophosphamide, intravenous immunoglobulin and rituximab) were also recorded.

Patients were divided in two groups with similar disease duration: cSLE patients with AIH (evaluated at AIH diagnosis) and cSLE patients without AIH (evaluated at last visit).

\section{Statistical analysis}

Results were presented as absolute number (frequency) for categorical variables and median (range) or mean \pm standard deviation for continuous variables. Categorical variables comparisons were assessed by Pearson $X$-Square or Fisher's exact test. Continuous variables from cSLE patients with and without AIH were compared by Mann-Whitney test or $\mathrm{t}$ test as appropriate. Statistical analysis was performed using Bonferroni correction $(p<0.0033)$.

\section{Results}

Demographic data, clinical and laboratorial features, outcomes and treatments in cSLE patients with $\mathrm{AIH}$ are described in Table 1 . AIH in cSLE patients confirmed by biopsy was observed in 7/847 (0.8\%) and all were diagnosed during adolescence. The majority occurred before or at cSLE diagnosis [5/7 (71\%)]. Antinuclear antibodies were a universal finding, 43\% had concomitantly anti-SMA and all were seronegative for anti-LKM-1 antibodies. All 7 patients with follow-up $\geq 18$ months (4/7) had complete response to therapy according to IAHGC. None of cSLE patients with AIH had severe hepatic manifestations such as hepatic failure, portal hypertension, cirrhosis or deceased at presentation or at follow-up (Table 1).

Liver biopsy was performed in only 7/847 (0.8\%) cSLE that fulfilled the AIH criteria (IAHGC) $[4,5]$. Regarding 
Table 1 Demographic data, clinical and laboratorial features, outcomes and treatments in childhood-onset systemic lupus erythematosus (CSLE) patients with autoimmune hepatitis (AlH)

\begin{tabular}{|c|c|c|c|c|c|c|c|}
\hline & \multicolumn{7}{|l|}{ Cases } \\
\hline & 1 & 2 & 3 & 4 & 5 & 6 & 7 \\
\hline \multicolumn{8}{|l|}{ Demographic data } \\
\hline Age, years & 12.5 & 12.3 & 10.3 & 15 & 10.3 & 15.6 & 11.7 \\
\hline Female gender & + & + & + & + & + & + & - \\
\hline Time between AlH and CSLE, years & -1.5 & 0 & -4.75 & 0 & 2.3 & 8.5 & 0 \\
\hline CSLE duration, years & 0 & 0 & 0 & 0 & 2.3 & 8.5 & 0 \\
\hline \multicolumn{8}{|l|}{ Clinical/laboratorial features } \\
\hline Constitutional symptoms & - & + & + & + & + & + & + \\
\hline Jaundice/ascites & $+/-$ & $-/-$ & $+/-$ & $+/-$ & $-/-$ & $+/-$ & $+/-$ \\
\hline Hepatomegaly/Splenomegaly & $-/-$ & $+/+$ & $-/-$ & $+/+$ & $+/-$ & $+/-$ & $+/+$ \\
\hline AST, IU/L & 93 & 652 & 35 & 345.7 & 245 & 4466 & 1260 \\
\hline$A L T, I U / L$ & 113 & 268 & 122 & 244.6 & 552 & 1411 & 949 \\
\hline GGT, IU/L & 258 & 604 & 91 & 51.2 & 613 & 69 & 606 \\
\hline Hypergammaglobulinemia & + & + & + & + & + & + & + \\
\hline ANA $>1: 80$ & + & + & + & + & + & + & + \\
\hline Anti-SMA > 1:80 & + & + & + & - & - & - & - \\
\hline Anti-LKM1 > 1:80 & - & - & - & - & - & - & - \\
\hline Viral hepatitis markers & - & - & - & - & - & - & - \\
\hline Alcohol intake & - & - & - & - & - & - & - \\
\hline Liver histology with interface hepatitis and lymphocytic infiltrates & + & + & + & + & + & + & + \\
\hline \multicolumn{8}{|l|}{ Outcomes } \\
\hline Liver failure/portal hypertension/cirrhosis & $-/-/-$ & $-/-/-$ & $-/-/-$ & $-/-/-$ & $-/-/-$ & $-/-/-$ & $-/-/-$ \\
\hline Death & - & - & - & - & - & - & - \\
\hline \multicolumn{8}{|l|}{ Therapy } \\
\hline AlH complete response therapy (> 18 months) & + & NP & + & NP & + & + & NP \\
\hline cSLE treatments & $P D, A Z A$ & $\mathrm{PD}, \mathrm{AZA}, \mathrm{AM}$ & $P D, A Z A, A M$ & $P D, A Z A$ & $P D, A Z A, A M$ & $P D, A Z A$ & PD \\
\hline
\end{tabular}

AST aspartate aminotransferase (normal value 15-40 IU/L), ALT alanine aminotransferase (normal value 10-40 IU/L), GGT gamma glutamyl transferase (normal value 3-25 IU/L), ANA antinuclear antibodies, anti-SMA - anti-smooth muscle antibody, anti-LKM1 - anti-liver kidney microsomal antibody type1, NP not possible, PD prednisone, $A Z A$ azathioprine, $A M$ antimalarial

the remaining $840 \mathrm{cSLE}$ patients without $\mathrm{AIH}, 86 \%$ patients were females. The median of age was 12 years $(0.25-18)$ and the median of disease duration was 5 years (0-23). Leukopenia (45\%), thrombocytopenia (21\%), ANA (99\%), anti-dsDNA antibodies (71\%) and anti-Sm antibodies (32\%) were observed in these cSLE patients.

Deaths occurred in 69/840 (8\%) cSLE patients that were not affected by AIH. Infections accounted for 54/69 (78\%) of overall deaths and $70 \%$ of these had concomitant disease activity. Other causes of death were: nephritis (acute kidney injury or chronic renal disease) (9\%), alveolar hemorrhage (4\%), massive intracranial bleeding (1.4\%), multiple thrombosis due to catastrophic antiphospholipid syndrome (1.4\%), B-cell lymphoma (1.4\%) and unknown etiologies (4\%).

Further analysis of 7 cSLE patients with AIH compared to 28 cSLE patients without AIH and with the same disease duration $[0(0-8.5)$ vs. $0.12(0-8.5)$ years, $p=0.06$ ] revealed that the frequency of hepatomegaly was significantly higher in CSLE patients with AIH compared to those without AIH ( $71 \%$ vs. $11 \%, p=0.003)$. The median of age at diagnosis [12.25 (10.3-15.6) vs. $12.9(3.3-19.7)$ years, $p=0.650]$ and SLEDAI-2 K score [6 (0-26) vs. $7(0-41)$ years, $p=0.755]$ were similar in both groups. No differences were evidenced between constitutional involvement, splenomegaly, serositis, musculoskeletal, neuropsychiatric and renal manifestations, and treatments in cSLE patients with and without $\mathrm{AIH}$ $(p>0.0033)$ (Table 2).

The comparisons of last visit of cSLE patients with AIH $(n=7)$ versus last visit of CSLE without AIH $(n=840)$ revealed similar age $[12.25(7.1-15)$ vs. $11.83(0.25-17.8)$ years, $p=0.94]$ and disease duration $[4.3(0.58-11.8)$ vs. $4.58(0-23.4)$ years, $p=0.56]$ in both groups. The frequency 
Table 2 Demographic data, disease activity, cumulative clinical manifestations and treatments in childhood-onset systemic lupus erythematosus (CSLE) patients with autoimmune hepatitis (AIH) at diagnosis compared to those without AlH evaluated at last visit

\begin{tabular}{|c|c|c|c|}
\hline Variables & $\begin{array}{l}\text { CSLE with AlH } \\
\text { (at AlH diagnosis) } \\
(n=7)\end{array}$ & $\begin{array}{l}\text { CSLE without AlH } \\
\text { (at last visit) } \\
(n=28)\end{array}$ & P \\
\hline \multicolumn{4}{|l|}{ Demographic data } \\
\hline Age at diagnosis, years & $12.25(10.3-15.6)$ & $12.9(3.3-19.7)$ & 0.650 \\
\hline Disease duration, years & $0(0-8.5)$ & $0.12(0-8.5)$ & 0.060 \\
\hline \multicolumn{4}{|l|}{ Disease activity parameter } \\
\hline SLEDAI-2 K & $6(0-26)$ & $7(0-41)$ & 0.755 \\
\hline \multicolumn{4}{|l|}{ Cumulative clinical manifestations } \\
\hline Constitutional involvement & $6(86)$ & $7(25)$ & 0.006 \\
\hline Hepatomegaly & $5(71)$ & $3(11)$ & $0.003^{*}$ \\
\hline Splenomegaly & $3(43)$ & $1(4)$ & 0.019 \\
\hline Musculoskeletal involvement & $4(57)$ & $3(11)$ & 0.018 \\
\hline Serositis & $0(0)$ & $4(7)$ & 1.000 \\
\hline Neuropsychiatric involvement & $0(0)$ & $5(18)$ & 0.559 \\
\hline Renal involvement & $2(29)$ & $11(39)$ & 0.689 \\
\hline \multicolumn{4}{|l|}{ Treatment } \\
\hline Prednisone dose, mg/kg/day & $1.07(0.5-2.4)$ & $0.9(0.1-2.8)$ & 0.365 \\
\hline Antimalarial use & $3(43)$ & $14(50)$ & 1.000 \\
\hline Immunosuppressive use & $6(86)$ & $8(29)$ & 0.009 \\
\hline
\end{tabular}

Results are presented in $\mathrm{n}(\%)$ and median (range), ${ }^{*} P$ - value according to Bonferroni correction for multiple comparisons $(p<0.0033)$, SLEDAI-2 K - SLE Disease Activity Index 2000

of female gender $(85.7 \%$ vs. $85.9 \%, p=1.0)$ and median of SLEDAI-2 K [4 (2-10) vs. $2(0-45), p=0.45]$ and SLICC-ACR/damage index $[0(0-1)$ vs. $0(0-9), p=$ 0.77] were also similar in both groups. The frequencies of chronic renal failure ( $0 \%$ vs. $6.1 \%, \mathrm{p}=1.0)$, prednisone $(100 \%$ vs. $97 \%, p=1.0)$, azathioprine ( $86 \%$ vs. $61 \%$, $p=0.26)$, methotrexate ( $29 \%$ vs. $23 \%, p=0.66)$, mycophenolate mofetil $(14 \%$ vs. $21 \%, p=1.0)$ and intravenous cyclophosphamide ( $0 \%$ vs. $42 \%, p=0.05$ ) were alike in both group. The lower frequency of death in AIH patients did not reach statistical significance $(0 \%$ vs. $8 \%$, $p=1.0)$.

The Kaplan-Meier overall survival curve was significantly higher in cSLE patients with $\mathrm{AIH}$ compared to those without this complication $(p=0.001)$. After 11.6 years of disease onset the survival percentage of cSLE patients with $\mathrm{AIH}$ was $100 \%$ and for those without $\mathrm{AIH}$ was $83 \%$ (Fig. 1).

\section{Discussion}

This multicenter study demonstrated that overlap of $\mathrm{AIH}$ and cSLE is a very rare association. This liver autoimmune disease occurred mainly at the first years of cSLE diagnosis, without liver complication and associated with mild disease manifestations.

The strength of this study was the large cohort including 10 different Pediatric Rheumatology and tertiary centers of
cSLE. All AIH patients fulfilled the IAHGC definite criteria (score $>15$ ) with typical histological features in liver biopsy $[4,5]$. We also used a standardized cSLE protocol with definitions for clinical and disease activity parameters [10]. The limitations were the possible missing data due to retrospective design, the collinearity of the variables and the limited indication for liver biopsy.

We have confirmed and extended previous observation that liver involvement is rarely observed in cSLE and adult SLE patients, with a variety of clinical and laboratorial manifestations [1, 12-16]. The rigorous selection criteria of AIH and cSLE patients excluding viral infections, malignancies and alcohol intake was relevant to avoid other confounding etiologies of hepatitis $[1,7,12,14]$.

$\mathrm{AIH}$ was solely observed in adolescents and the majority occurred before or at cSLE diagnosis. Hepatomegaly was a distinctive feature of $\mathrm{AIH}$ in this age group contrasting with our previous observation that liver enlargement was usually not common in adolescent at lupus diagnosis [17]. Hepatomegaly was not associated with liver congestion, fatty infiltration, viral hepatitis, metabolic disorders, thrombosis or hepatotoxic drugs usage, since histological findings excluded these issues. The concomitant presences of jaundice, splenomegaly and hypergammaglobulinemia reinforced the AIH diagnosis [1], as observed in more than $50 \%$ of cases.

AIH occurred in cSLE patients with mild lupus manifestations. Of note, the majority of our patients had a 


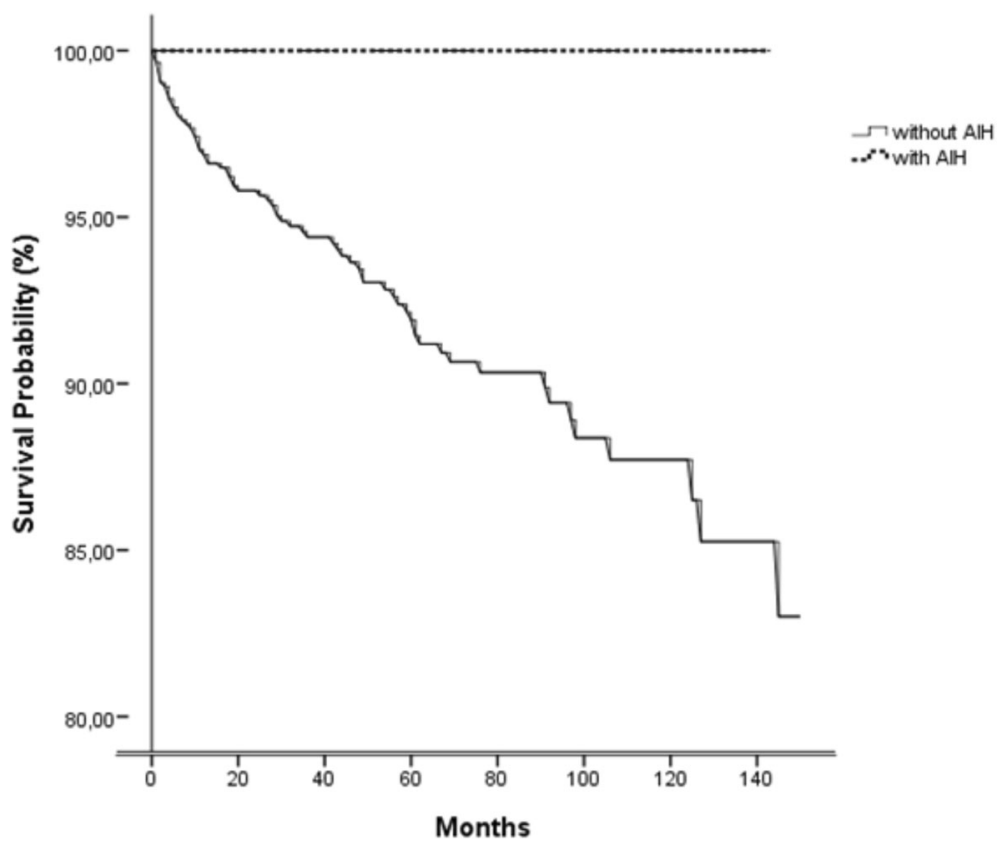

Fig. 1 Kaplan-Meier overall survival curve in childhood-onset systematic lupus erythematosus patients with autoimmune hepatitis (AlH) compared to those without this complication

complete response to the classical AIH prednisone and azathioprine combination therapy. Reinforcing this finding none of our patients had hepatic failure, portal hypertension and cirrhosis suggesting that AIH phenotype in lupus was a non-severe pattern $[1,3]$. Additionally, none of our cSLE patients had anti-LKM1 antibodies.

\section{Conclusion}

Overlap AIH and cSLE was rarely observed in this large multicenter study and hepatomegaly was the distinctive clinical feature of these patients. AIH occurred during adolescence, mainly at the first years of lupus and it was associated with mild liver manifestations.

\section{Acknowledgements}

Our gratitude to Ulysses Doria-Filho for the statistical analysis. The authors thank the following Pediatric Rheumatology Divisions and colleagues for including their patients: Pediatric Rheumatology Unit, Children's Institute, FMUSP (Adriana Maluf Elias Sallum, Cristina Miuki Abe Jacob, Gabriela Blay, Gabriela Nunes Leal, Heloisa Helena de Souza Marques, João Domingos Montoni da Silva, Joaquim Carlos Rodrigues, Juliana Caíres de Oliveira Achili Ferreira, Kátia Kozu, Laila Pinto Coelho, Luciana dos Santos Henriques, Magda Carneiro-Sampaio, Maria Helena Vaisbich, Roberta Cunha Gomes, Victor Leonardo Saraiva Marques, Werther Brunow de Carvalho); Pediatric Rheumatology Unit, UNIFESP (Anandreia Simões Lopes, Claudio Len, Daniela Petry Piotto, Giampaolo Faquin, Gleice Clemente, Maria Odete Esteves Hilário, Melissa Fraga, Octavio Augusto Bedin Peracchi, Vanessa Bugni); Division of Rheumatology, FMUSP (Juliane A Paupitz, Glauce L Lima); UNESP (Priscila R. Aoki, Juliana de Oliveira Sato, Silvana Paula Cardin, Taciana Albuquerque Pedrosa Fernandes), Irmandade da Santa Casa de Misericórdia de São Paulo (Andressa Guariento, Eunice Mitiko Okuda, Silvana Brasília Sacchetti), State University of Campinas (Simone Appenzeller, Renata Barbosa, Roberto Marini), Ribeirão Preto Medical School - University of São Paulo (Francisco Hugo Gomes, Gecilmara Salviatto Pileggi, Luciana Martins de Carvalho, Paola Pontes Pinheiro), Hospital Infantil
Darcy Vargas (Cássia Maria Passarelli Lupoli Barbosa, Luciana Tudech Pedro Paulo), Pontifical Catholic University of Sorocaba (Valeria Cristina Santucci Ramos); Hospital Municipal Infantil Menino Jesus (Simone Lotufo and Tânia Caroline Monteiro Castro).

\section{Funding}

This study was supported by grants from Conselho Nacional de Desenvolvimento Científico e Tecnológico (CNPq 303422/2015-7 to CAS), Fundação de Amparo à Pesquisa do Estado de São Paulo (FAPESP 2015/ 03756-4 to (AS), Federico Foundation (to CAS) and by Núcleo de Apoio à Pesquisa "Saúde da Criança e do Adolescente" da USP (NAP-CriAd) to CAS.

\section{Availability of data and materials Not applicable}

\section{Authors' contributions}

$V A B, B M, A C P, A R S, L P C, J C O F, R M R P, M T T, C S M, N E A, A P S, K K, L M C, A M S$, VPF, DPP, EB and CAS analyzed and interpreted the patient data regarding autoimmune hepatitis in childhood onset systemic lupus erythematosus. $V A B, B M, A C P, E B$ and $C A S$ were the major contributor in writing the manuscript. All authors read and approved the final manuscript.

Ethics approval and consent to participate

This study was approved by our Ethics Committee.

\section{Consent for publication}

Not applicable

\section{Competing interests}

The authors declare that they have no competing interests.

\section{Publisher's Note}

Springer Nature remains neutral with regard to jurisdictional claims in published maps and institutional affiliations. 


\section{Author details}

'Pediatric Rheumatology Unit, Children's Institute, Hospital das Clinicas HCFMUSP, Faculdade de Medicina, Universidade de Sao Paulo, Sao Paulo, SP, Brazil. ${ }^{2}$ Division of Rheumatology, Hospital das Clinicas HCFMUSP, Faculdade de Medicina, Universidade de Sao Paulo, Sao Paulo, SP, Brazil. ${ }^{3}$ Pediatric Rheumatology Unit, Universidade Federal de São Paulo, São Paulo, Brazil. ${ }^{4}$ Pediatric Rheumatology Unit, São Paulo State University (UNESP) Faculdade de Medicina de Botucatu, São Paulo, Brazil. ${ }^{5}$ Pediatric Rheumatology Unit, Ribeirão Preto Medical School, University of São Paulo, São Paulo, Brazil.

Received: 22 February 2018 Accepted: 3 December 2018

Published online: 13 December 2018

\section{References}

1. Deen ME, Porta G, Fiorot FJ, Campos LM, Sallum AM, Silva CA. Autoimmune hepatitis and juvenile systemic lupus erythematosus. Lupus. 2009;18:747-51.

2. Aikawa NE, Jesus AA, Liphaus BL, Silva CA, Carneiro-Sampaio M, Viana VS, et al. Organ-specific autoantibodies and autoimmune diseases in juvenile systemic lupus erythematosus and juvenile dermatomyositis patients. Clin Exp Rheumatol. 2012;30:126-31.

3. Sönmez HE, Karhan AN, Batu ED, Bilginer $Y$, Gümüş E, Demir $H$, et al Gastrointestinal system manifestations in juvenile systemic lupus erythematosus. Clin Rheumatol. 2017;36:1521-6.

4. Alvarez F, Berg PA, Bianchi FB, Bianchi L, Burroughs AK, Cancado EL, et al. International autoimmune hepatitis group report: review of criteria for diagnosis of autoimmune hepatitis. J Hepatol. 1999;31:929-38.

5. European Association for the Study of the Liver. EASL clinical practice guidelines: autoimmune hepatitis. J Hepatol. 2015;63:971-1004.

6. Battagliotti C, Rispolo Klubek D, Karakachoff M, Costaguta A. An overlap syndrome involving lupus erythematosus and autoimmune hepatitis in an adolescent girl. Arch Argent Pediatr. 2016;114:155-8.

7. Alves SC, Fasano S, Isenberg DA. Autoimmune gastrointestinal complications in patients with systemic lupus erythematosus: case series and literature review. Lupus. 2016;25:1509-19.

8. Lopes SRM, Gormezano NWS, Gomes RC, Aikawa NE, Pereira RMR, Terrer MT, et al. Outcomes of 847 childhood-onset systemic lupus erythematosus patients in three age groups. Lupus. 2017;26:996-1001.

9. American College of Rheumatology. 1997 update of the 1982 American College of Rheumatology revised criteria for classification of systemic lupus erythematosus. Arthritis Rheum. 1997;40:1725.

10. Gladman DD, Ibañez D, Urowitz MB. Systemic lupus erythematosus disease activity index 2000. J Rheumatol. 2002;29:288-91.

11. American College of Rheumatology Ad Hoc committee on neuropsychiatric Lupus Syndromes. The American College of Rheumatology nomenclature and case definitions for neuropsychiatric lupus syndromes. Arthritis Rheum. 1999;42:599-8

12. Irving KS, Sen D, Tahir H, Pilkington C, Isenberg DA. A comparison of autoimmune liver disease in juvenile and adult populations with systemic lupus erythematosus-a retrospective review of cases. Rheumatology. 2007:46:1171-3.

13. Silva CA. Childhood-onset systemic lupus erythematosus: early disease manifestations that the paediatrician must know. Expert Rev Clin Immunol. 2016;12:907-10.

14. Guariento A, Silva MF, Tassetano PS, Rocha SM, Campos LM, Valente M, et al. Liver and spleen biometrics in childhood-onset systemic lupus erythematosus patients. Rev Bras Reumatol. 2015;55:346-51.

15. Lim DH, Kim YG, Lee D, Min Ahn S, Hong S, Lee CK, et al. Immunoglobolin G levels as a prognostic factor for autoimmune hepatitis combined with systemic lupus erythematosus. Arthritis Care \& Res (Hoboken). 2016;68:995-1002.

16. Teufel A, Weinmann A, Kahaly GJ, Centner C, Piendl A, Wörns M, et al. Concurrent autoimmune diseases in patients with autoimmune hepatitis. J Clin Gastroenterol. 2010;44:208-13.

17. Gomes RC, Silva MF, Kozu K, Bonfá E, Pereira RM, Terreri MT, et al. Features of 847 Childhood-Onset Systemic Lupus Erythematosus Patients in Three Age Groups at Diagnosis: A Brazilian Multicenter Study. Arthritis Care Res (Hoboken). 2016;68:1736-41.

Ready to submit your research? Choose BMC and benefit from:

- fast, convenient online submission

- thorough peer review by experienced researchers in your field

- rapid publication on acceptance

- support for research data, including large and complex data types

- gold Open Access which fosters wider collaboration and increased citations

- maximum visibility for your research: over $100 \mathrm{M}$ website views per year

At BMC, research is always in progress.

Learn more biomedcentral.com/submissions 